\title{
A REPORT ON RUSSIAN MAGNET TECHNOLOGY FOR USE IN PHI FACTORY AND LIGHT SOURCE BENDING MAGNETS
}

\author{
M. A. Green \\ Law rence Berkeley Laboratory \\ University of California \\ Berkeley, CA 94720
}

*This work was performed at the Lawrence Berkeley Laboratory with the support of the Office of Astrophysics, NASA and the Director of the Office of Energy Research, Office of High Energy and Nuclear Physics, High Energy Physics Division, U. S. Department of Energy under Contract No. DE-AC03-76SF00098. 


\title{
A Report on Russian Magnet Technology for Use in Phi Factory and Light Source Bending Magnets
}

\author{
Michael A. Green \\ Lawrence Berkeley Laboratory \\ Berkeley CA. 94720
}

Short superconducting dipole magnets are a key element for the UCLA phi factory and for the phi factory proposed by the Institute of Physics at Novosibirsk Siberia. The requirements for these short dipole magnets are as follows:

1. The central field of the dipole has to be between 4 and 6 tesla in order that the dipole field provide damping as well as bending. If conventional 1.5 tesla dipole magnets were used, one would have to add about 10 meters of conventional rare earth permanent wiggler magnets in order to provide the damping need to insure a small beam spot size at the interaction region. The concept of adding 10 meters wiggler to provide damping is inconsistent with the small ring philosophy which is part of both the UCLA and the Novosibirsk phi factory ring design concepts.

2) The dipole magnet bore has to be open so that the synchrotron light can exit from the bending magnet. In addition space is required for vacuum pumping in the magnet bore.

3) The magnets have to have a relatively low current so that the magnet electrical leads do not use much helium refrigeration. This issue is particularly important because the dipole magnets are small and widely separated. Unlike the SSC dipoles, these dipoles can not share a common cryostat.

Compact synchrotron dipole magnet designs from American, English and Japanese companies do not meet the requirements of either the UCLA or the Novosibirsk phi factories. In all of the compact synchrotron dipole designs, iron is not used to return the magnetic flux. Stray field at the ends of the dipole magnets is a continuing problem to the machine designer with any of the three compact synchrotron magnet design concepts. The compact synchrotron magnets which have been proposed and or built by these companies provide 180 degrees of bending in a single magnet. Because the bend is 180 degrees, the dipole has to provide focusing as well. The only really successful compact synchrotron dipole which has been developed is the one from Oxford Magnet Technology in England. The Oxford compact synchrotron costs between 20 and 25 million dollars and it is not suitable for doing phi physics.

If one is willing to make the machine circumference larger than the compact synchrotron built by Oxford, one can put conventional quadrupole magnets between the bending magnets which bend the beam from 45 to 60 degrees in each magnet. No satisfactory short bend dipole magnet has emerged from university, national laboratory or industrial sources in the United States. However, there is a very good short dipole design which has been developed by the Pavel Vobly of the Institute for Nuclear Physics in Novosibirsk. The Vobly dipole magnet has a number of very attractive characteristics which are as follows: 
1. The central induction of the dipole can be between 2 tesla and 6 tesla and the field uniformity can be better than one unit (1 part in 10000) over the aperture required for the particle beam. The two current version of the Vobly design permits one to tune the magnet so that good field can be obtained over a whole range of central inductions.

2. The Vohly dipoles are relatively short with a magnetic length between 300 and 500 millimeters. The dipole magnet can be made so that its physical length (the warm length) is less than 50 millimeters longer than its magnetic length.

3. The Vobly dipole design carries all of its return flux in iron so that the magnetic flux leakage is minimized. The field profile at the ends of the dipole is very similar to that of a conventional 1.5 to 1.8 tesla dipole. The stray field from a Vobly dipole should have a smaller effect on the machine beam dynamics. It is probable that the best design would be a cold i, design. Pavel Vobly claims that his design can have mixed both cold and warm iron.

4. The Vobly magnet horizontal good field aperture can be made to be two or three times the gap between the iron poles. This means that there is room for beam sagita and there is room for the kind of vacuum chamber needed in a phi factory or a synchrotron light source. Since the dipole is short and its horizontal aperture is broad, there is room for most of the synchrotron radiation to escape the magnet. For this reason, the Vobly magnet design is well suited for a compact light source.

5. The Vobly dipole magnet can be made with eight strand cable or a monolithic conductor with an aspect ratio of four to one. Because the conductor is relatively small, the current in the magnet can be made to be relatively low compared to a smaller aperture SSC dipole magnet.

6. The Vobly type dipoles can be fabricated in either straight configuration or a curved segment configuration. In either configuration the Vobly design behaves magnetically like a low field conventional copper iron dipole.

7. The Vobly magnet can be fabricated for less money than other designs. Our estimates indicate that a single Vobly phi factory dipole can be fabricated for about a half a million dollars. The group at Novosibirsk has quoted a price of one hundred thousand US dollars for a single 60 degree phi factory dipole.

The proposed 60 degree dipoles for the Novosibirsk phi factory are straight, so there will be focusing off of the edges of the magnet. Parameters of the proposed Novosibirsk phi factory dipole magnet based on the Vobly design is shown in Table 1. A cross-section of the Novosibirsk phi factory magnet is shown in Figure 1. The Vobly magnet design shown in Table 1 and Figure 1 can be scaled directly for use in a phi factory at UCLA. The symmetric UCLA phi factory magnet design called for a 4.0 tesla dipole which had an aperture of about $170 \mathrm{~mm}$ and a cold gap of about $70 \mathrm{~mm}$. This proposed UCLA dipole magnet was a 45 degree bending magnet with a magnetic length of just over $308 \mathrm{~mm}$. This magnet would fit into the same general physical envelope of the magnet described in Table 1. 


\section{Table I Parameters for the Novosibirsk Superconducting Phi Factory Dipole Magnet}

$\begin{array}{lc}\text { Dipole Overall Length }(\mathrm{mm}) & 410 . \\ \text { Dipole Overall Height }(\mathrm{mm}) & \sim 720 . \\ \text { Dipole Overall Width (mm) } & \sim 720 . \\ \text { Dipole Magnetic Length (mm) } & 330 . \\ \text { Dipole Horizontal Aperture (mm) } & 140 . \\ \text { Dipole Vertical Gap Aperture (mm) } & 56 . \\ \text { One Unit* Good Field Horizontal Aperture (mm) } & \sim 98 . \\ \text { One Unit* Good Field Vertical Aperture (mm) } & \sim 45 \\ \text { Beam Vacuum Pipe Diameter (mm) } & 40 . \\ \text { Dipole Magnet Cold mass (kg) } & \sim 1000 . \\ \text { Magnet Design Current (A) } & 2380 . \\ \text { Magnet Central Induction at Design Current (T) } & 5.15 \\ \text { Peak Induction in the Windings (T) } & \sim 5.7 \\ \text { Dipole Self Inductance (H) } & 0.0395 \\ \text { Dipole Stored Energy at its Design Current }(\mathrm{kJ}) & 112 .\end{array}$

* One unit of field error is 1 part in 10000

The Vobly magnet design concept has been extended to a number of other types of dipole magnets. Some examples of the other types of Vobly magnets include the following; 1 ) C type magnets which are segments where all of the synchrotron light escapes the bore, 2) a 10 tesla LHC superferric $60 \mathrm{~mm}$ aperture dipole magnet, 3) superconducting wigglers and undulators for use in a storage ring, and 4) a 90 degree bending magnet for use in a compact light source (See Figure 2). The Vobly magnet design concept is well suited for short dipole magnets where the amount of superconductor in the magnet is not an important cost factor. For short phi factory bending magnets, the Vobly magnet design is the best of those which have been under consideration. The Vobly magnets described here are also well suited for use in a compact light source.

\section{ACKNOWLEDGEMENTS}

The author acknowledges the discussions he has had with Pavel Vobly during a visit to the Institute for Nuclear Physics, Novosibirsk, Russia during the first week of June 1992. This work was supported by the Director of the Office of Energy Research, Office of High Energy and Nuclear Physics, United States Department of Energy under contract number DE-AC0376 SF00098.

\section{REFERENCES}

1. Michael A. Green, "Foreign Travel Report June 1992", concerning travel to Russia, the Ukraine, Lawrence Berkeley Laboratory Report LBID-1891, July 1992

2. P. Vobly, "A Brief Description of the Superconducting Bending Magnet with Parallel Faces for the Phi Factory Project", This report is unpublished and it describes the Vobly phi factory magnet design concept, (not dated) 


\section{Figure 1 A Cross-section View of a Vobly H Type Straight}

Dipole for Application as a Phi Factory Dipole

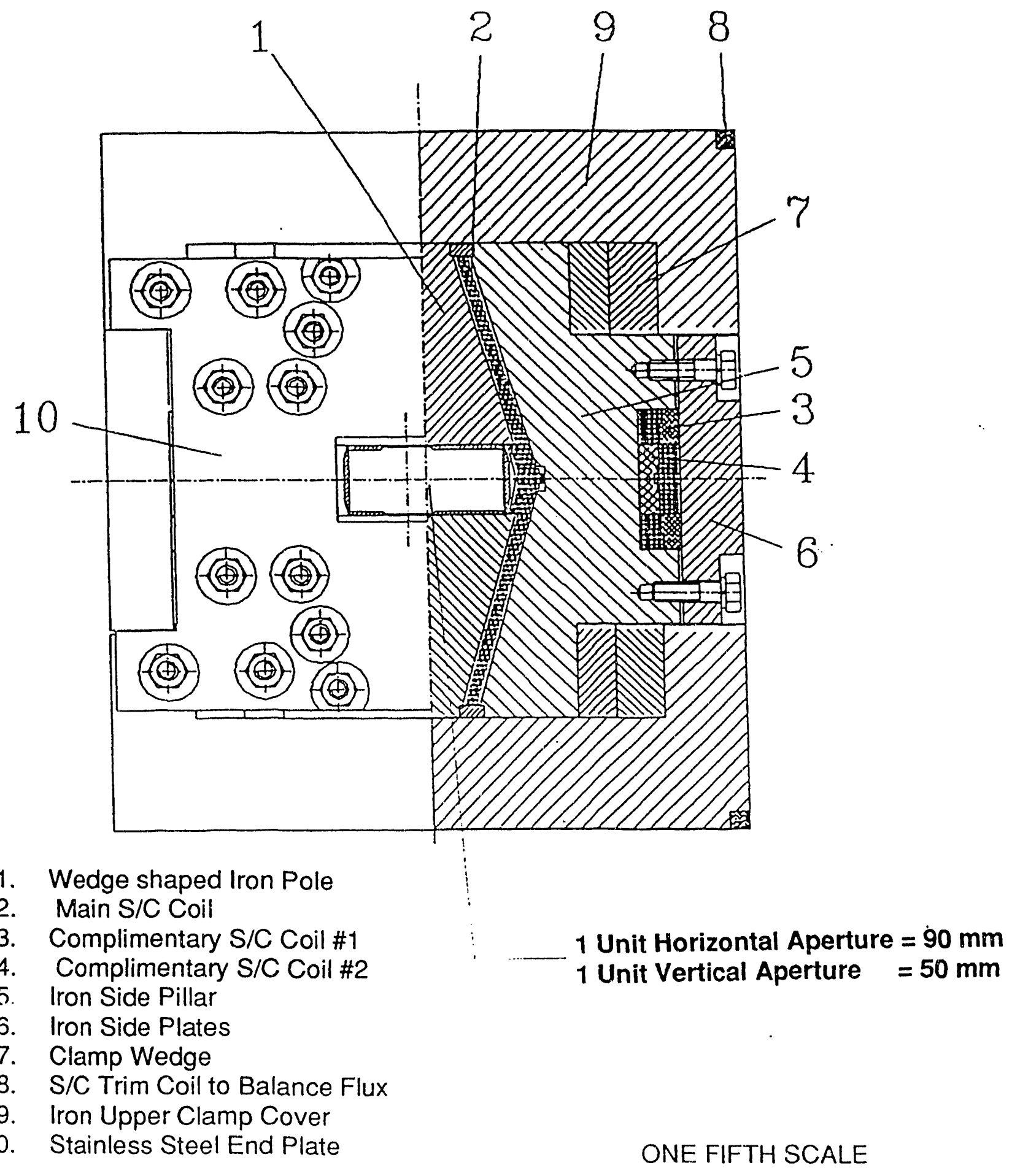

Not Shown: The Iron Return Path 


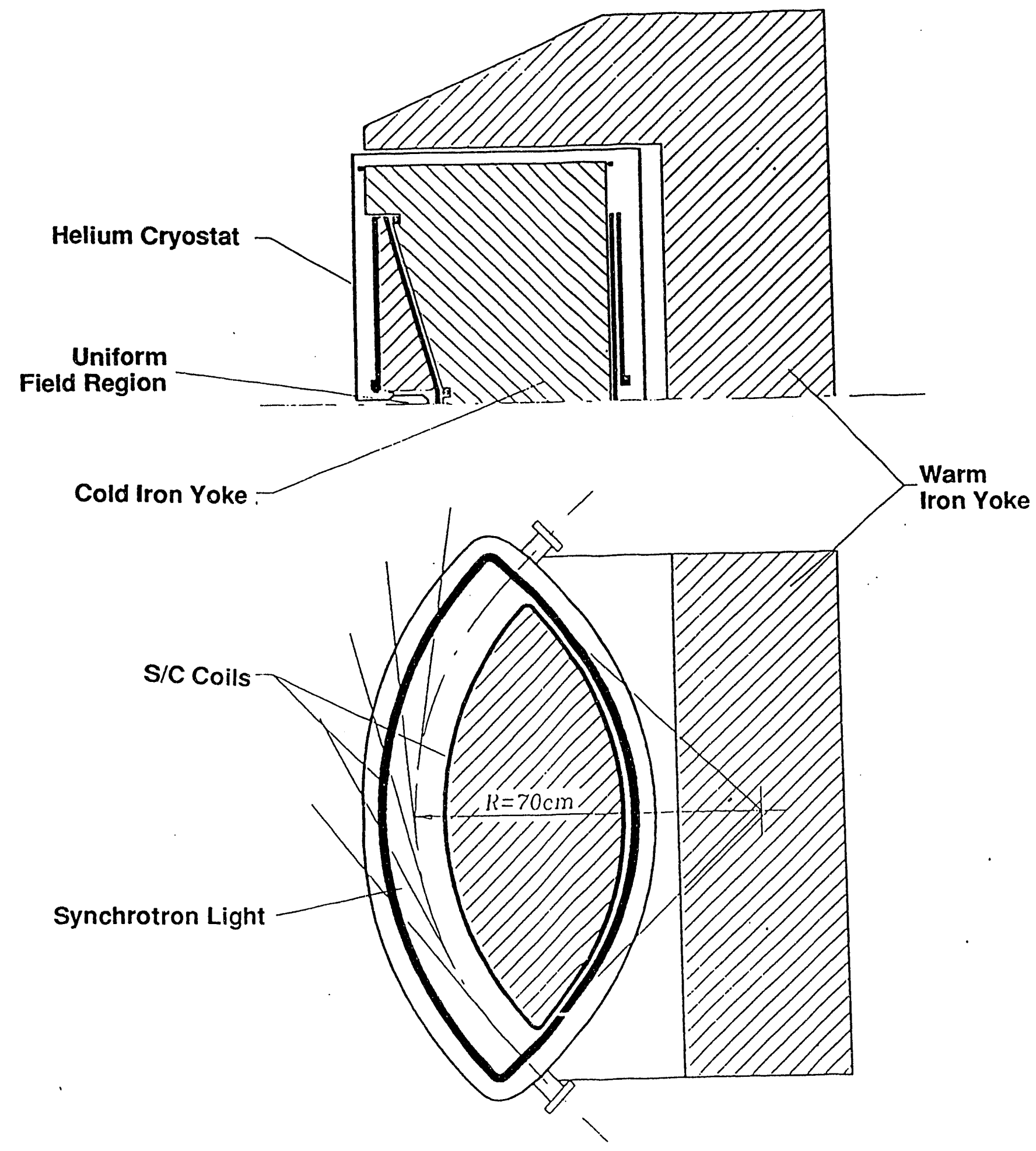

Figure 2 The Vobly 90 Degree C Type Bending Magnet with a Warm Iron Return (This magnet is well suited for a compact light source synchrotron.) 


$$
\text { " }
$$
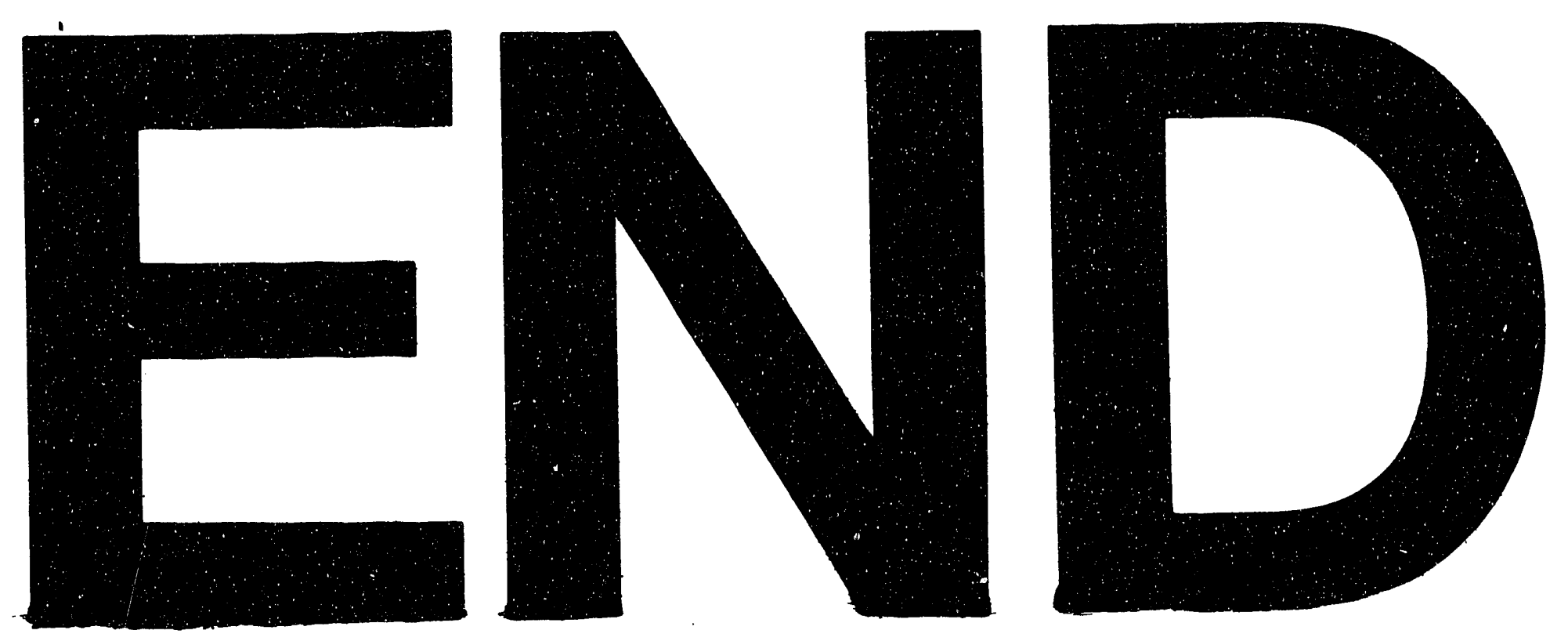

$x$
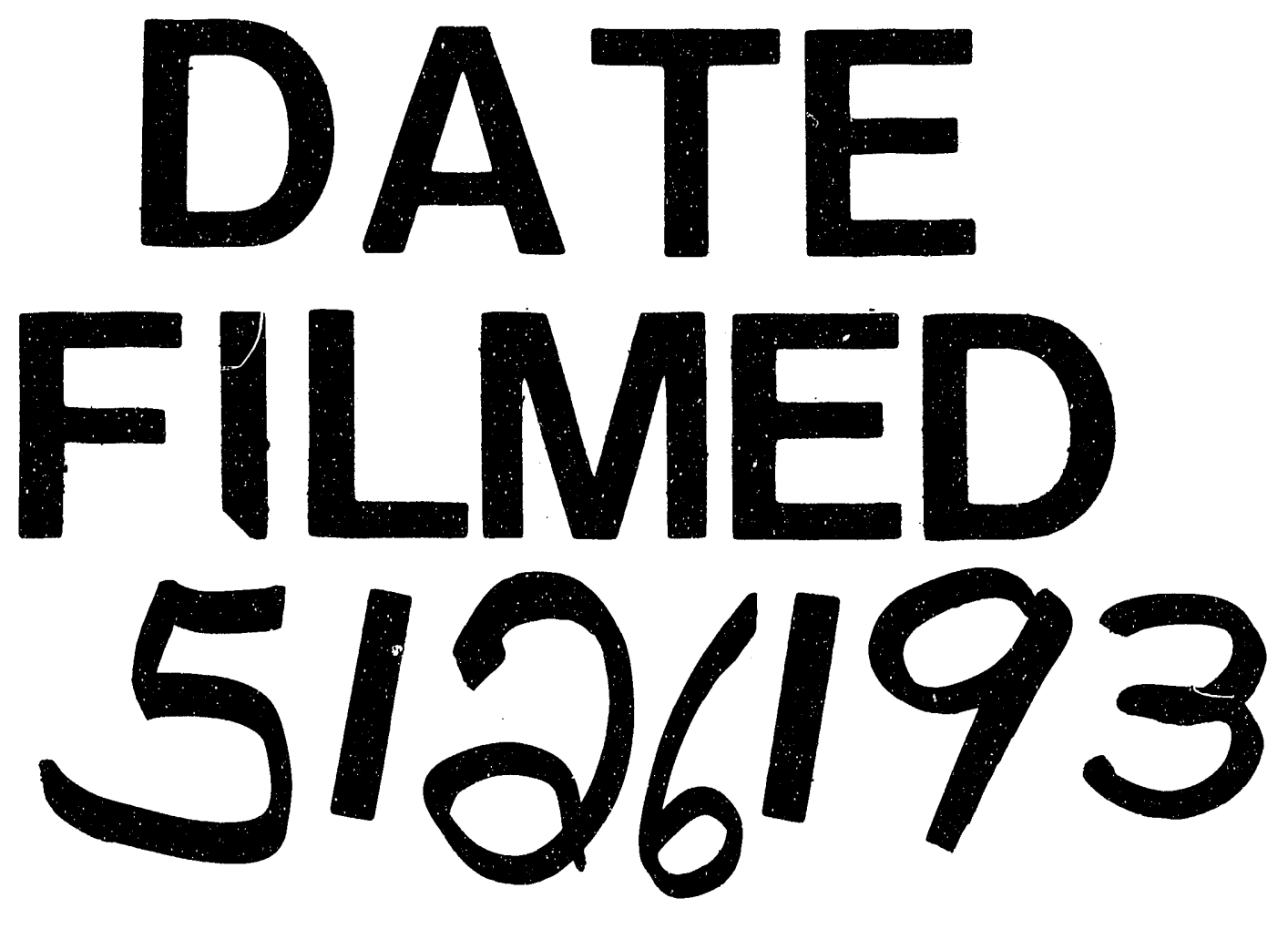
